\title{
Dispersion of Switching Processes in Ferroelectric Ceramics
}

\author{
O. V. Malyshkina, Anton Yurievich Eliseev, and R. M. Grechishkin
}

Tver State University, Sadovy Per. 35, Tver 170002, Russia

Correspondence should be addressed to O. V. Malyshkina; olga.malyshkina@mail.ru

Received 21 March 2017; Accepted 30 May 2017; Published 3 July 2017

Academic Editor: Charles Rosenblatt

Copyright (C) 2017 O. V. Malyshkina et al. This is an open access article distributed under the Creative Commons Attribution License, which permits unrestricted use, distribution, and reproduction in any medium, provided the original work is properly cited.

\begin{abstract}
The influence of the switching processes on self-heating of ferroelectric PZT ceramics samples was studied in high-amplitude sine and meander electric fields in a wide frequency range of 50 to $1500 \mathrm{~Hz}$. It is shown that the linear dependence of the self-heating temperature on the electric field frequency is observed only in low-frequency region. It was found that there exists a maximum on the frequency dependence of the self-heating temperature. The critical frequency $f_{\mathrm{cr}}$ corresponding to this maximum depends on both the properties of the material and geometry of the sample.
\end{abstract}

\section{Introduction}

Due to favourable combination of piezoelectric properties and coefficients of electromechanical coupling, the ferroelectric ceramics of lead zirconate titanate family (PZT) is the main functional material for the production of microelectromechanical systems (MEMS) [1-3]. Nevertheless piezoceramic elements for MEMS suffer from a number of disadvantages becoming apparent at high power regimes of working in high electric field strengths. Dictated by the nowadays increase of industrial applications of piezoelectric actuators a great attention is paid to fundamental studies of the problems of their reliability $[4,5]$. The main drawbacks of the piezoelectric material performance are connected to the existence of different kinds of losses.

The losses are generally classified into three main types: dielectric, mechanical, and electromechanical ones [4, 6]. Dielectric losses are associated with the hysteresis in the spontaneous polarization switching in alternating electric fields (dielectric hysteresis loop). Mechanical losses are characteristic of such applications of piezoceramics as ultrasound motors [7-9]. The higher they are the lower the mechanical figure of merit $Q_{m}$ is. In the resonance mode followed by the increase of vibration $[4,10-12]$ these losses lead to the rise of excess heat build-up related to field-induced mechanical stresses. Electromechanical losses as described by $[4,13]$ appear due to transformation of the electric energy (electric displacement $\mathbf{D}$, polarization $\mathbf{P}$ ) into mechanical deformation (stresses) owing to piezoelectric effect.

The authors of [4] mark out the following four causes of dielectric and electromechanical losses: (1) related to domain wall motion, (2) point crystal lattice defects, (3) microstructural losses at the grain boundaries because of material crystallinity, and (4) ohmic losses observed mainly in materials with high large electric conductivity. In piezoceramic materials the losses of the first kind dominate over other ones. They are related to the motion of domain walls including dielectric, elastic, and electromechanical hysteresis losses [3]. As a result of the losses significant undesirable selfheating comes into existence $[9,14,15]$.

The current theoretical understanding and experimental characterization of the complex self-heating processes in piezoelectric ceramics are far from being complete. In the present work we focus our attention on the study of selfheating and switching processes of piezoelectric elements in large AC electric fields of different frequency and waveform.

\section{Experiment}

A study is made of the polarization processes in PZT ceramics by AC sine and meander electric fields with an amplitude of 500 to $2100 \mathrm{~V} / \mathrm{mm}$ in a frequency range of 50 to $1500 \mathrm{~Hz}$. 


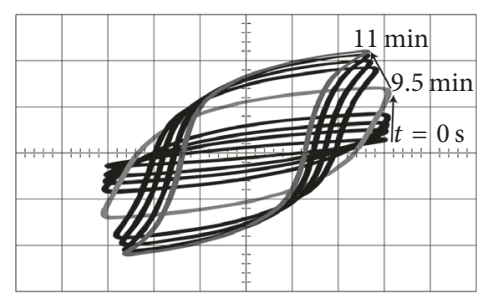

(a)

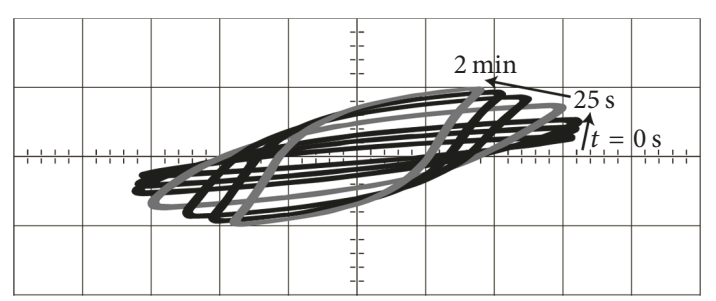

(b)

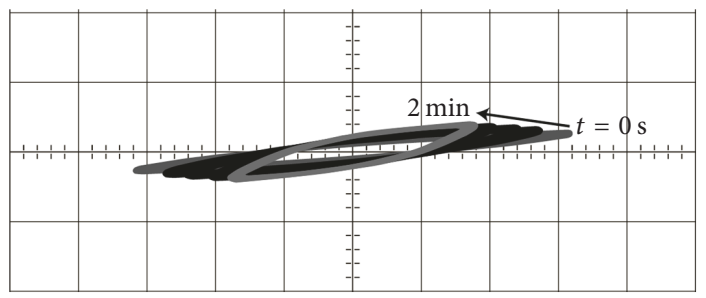

(c)

FIGURE 1: Evolution of dielectric hysteresis loops of PZT ceramics exposed to AC sine field at the frequencies 220 (a), 600 (b), and $1500 \mathrm{~Hz}$ (c). $E_{m}=840 \mathrm{~V} / \mathrm{mm}$, sample area $25 \mathrm{~mm}^{2}$.

Dielectric hysteresis loops were measured by the SawyerTower method simultaneously with distant temperature control with the aid of thermal vision camera Testo-875-1. High voltage amplifier TREK 677B was exploited as a source of AC voltage. The experimental results given below were obtained with square $(5 \times 5 \mathrm{~mm})$ or circular $(\varnothing 25 \mathrm{~mm})$ samples with a thickness of $1 \mathrm{~mm}$.

On applying an AC electric field of fixed amplitude, the samples initially demonstrate nonsaturated minor hysteresis loops that change their shape during further exposure under field (Figure 1). This reshaping is accompanied by a selfheating of the sample. The performed measurements have shown that the self-heating maximal temperature $\left(T_{\max }\right)$ depends not only on the applied field amplitude [16, 17], but also on its frequency (Figure 2). Increasing the sample area also results in an increase of $T_{\max }$. The latter also increases when the sine field excitation is replaced by a meander one (Figure 3).

Loop saturation was observed only for those frequency values $(220 \mathrm{~Hz}$ and above) for which the sample heating temperature exceeds $95^{\circ} \mathrm{C}$. It is this transition from the minor to full loop which corresponds to a sharp change of the sample temperature (Figure 2). This temperature range corresponds to a sharp decrease of the coercive field of the PZT which may be observed by ordinary heating of the sample in an oven. The rate of self-heating increases with the increase of the frequency thus resulting in the shortening of the time of hysteresis loop reshaping.

Dependence of the maximum temperature of the sample, $T_{\text {max }}$, on the applied field frequency is presented in Figure 4. It is seen that $T_{\max }$ of the sample with an area of $25 \mathrm{~mm}^{2}$ was exposed to sine field with $E_{m}=840 \mathrm{~V} / \mathrm{mm}$ at $220-250 \mathrm{~Hz}$. Further increasing of the frequency results in a decrease of $\left(T_{\max }\right)$; in doing so the area of the full loop also decreases (Figure 5(a)). By contrast, the hysteresis loop opening in

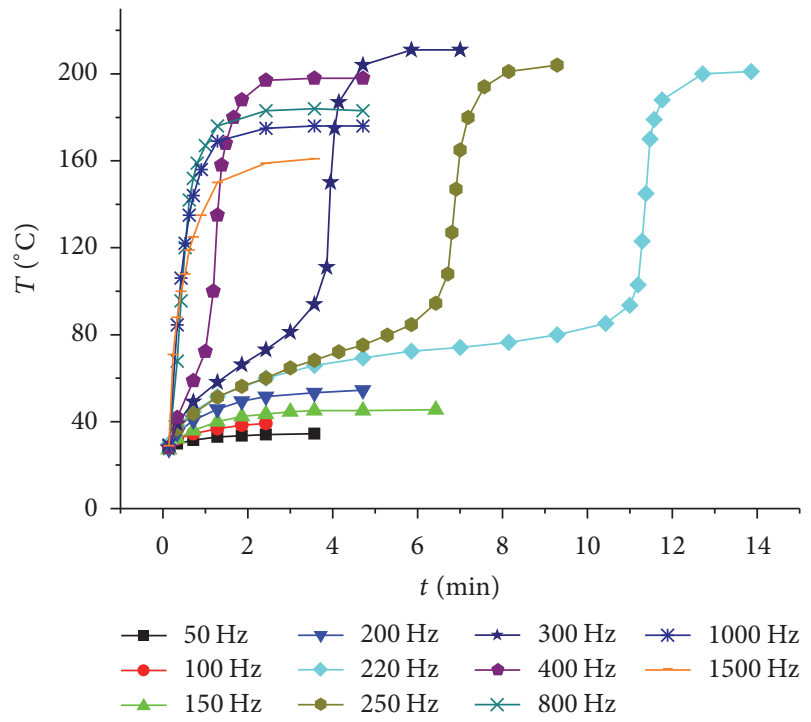

Figure 2: Time dependence of the temperature of the PZT sample with an area of $25 \mathrm{~mm}^{2}$ exposed to sine field of different frequencies.

meander field of the same amplitude as that of sine one is already observed at $150 \mathrm{~Hz}$ (Figure 5(b)).

\section{Results and Discussion}

The performed measurements show that the increase of the electric field frequency results in a decrease of reversible polarization values $\left(P_{r}\right)$ for both initial minor and reshaped full loops (Figure 5). In the last case, when the frequency corresponds to hysteresis loop saturation, the voltage drop occurs (Figure 1) which is explained by the increase of the conductivity. 


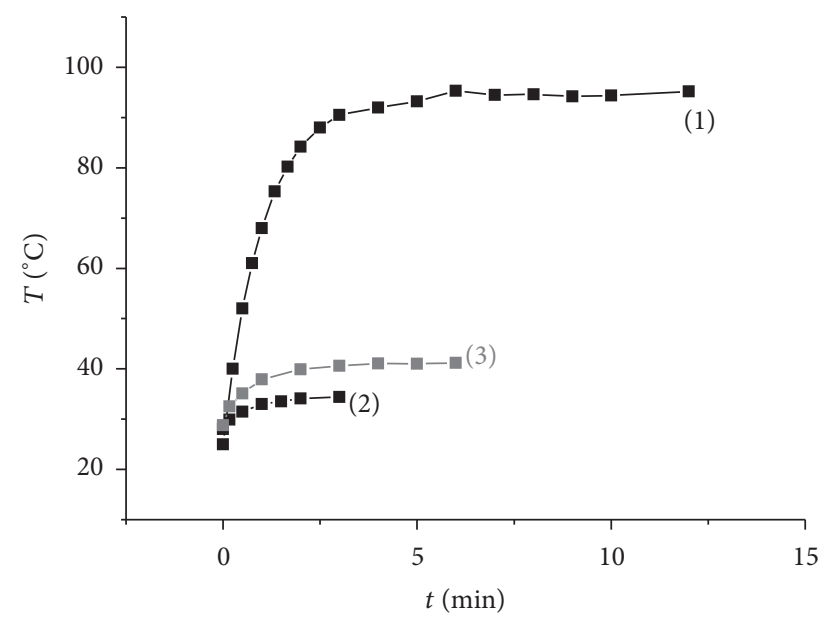

Figure 3: Time dependence of the temperature of the PZT sample of different area $S=490 \mathrm{~mm}^{2}$ (curve (1)) and $S=25 \mathrm{~mm}^{2}$ (curve (2)). Curves (1) and (2): sine wave excitation; curve (3): meander. $E_{m}=840 \mathrm{~V} / \mathrm{mm} . f=50 \mathrm{~Hz}$.

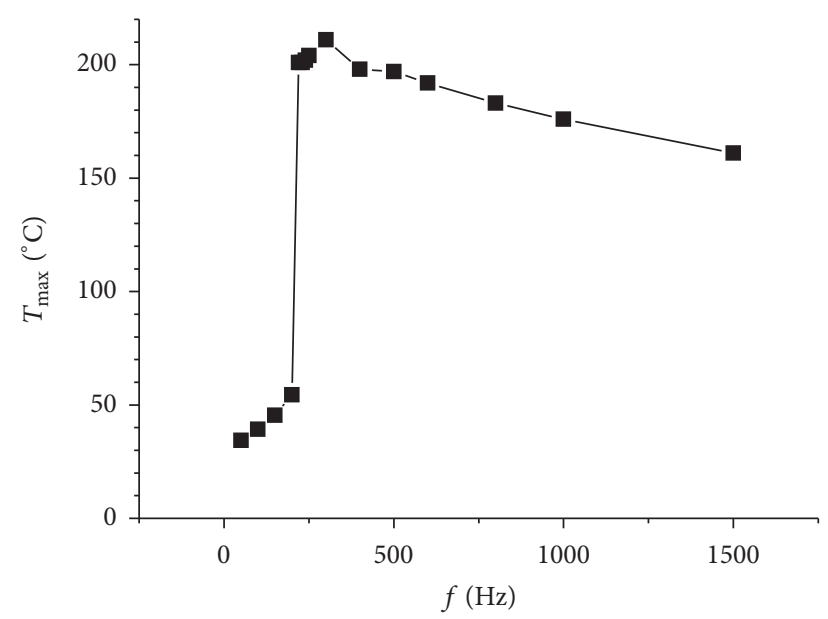

FIGURE 4: Dependence of the maximum self-heating temperature on the frequency of sine wave field. $E_{m}=840 \mathrm{~V} / \mathrm{mm}$, sample area $25 \mathrm{~mm}^{2}$.

It should be mentioned that when the sample is switched by meander voltage (Figure 5(b)), the magnitude of the switched polarization is larger compared with sine wave excitation (Figure 6, curves (1), (2)), other conditions being equal. In the case of meander, the loop opening begins at higher frequencies (Figure 6, curves (2)-(4)). At the same time, the magnitude of the switched polarization is independent of frequency. In all cases the decrease of the switched polarization value obeys the exponential rule independently of the frequency and shape of the loop after its opening (Figure 6).

Approximation of the experimental data making use of exponential regression with the aid of MathCad 14 resulted in an analytical expression for the frequency dependence of the polarization value $P$ :

$$
P=P_{s} \cdot \exp (-f \cdot \tau)+P_{\text {irr }} .
$$

TABLE 1: The coefficients of (1).

\begin{tabular}{lccc}
\hline & $P_{s}, \mathrm{C} / \mathrm{m}^{2}$ & $\tau, 10^{-3} \mathrm{~s}$ & $P_{\text {irr }}, \mathrm{C} / \mathrm{m}^{2}$ \\
\hline Sine & $0.28 \pm 0.01$ & $2.83 \pm 0.01$ & $0.03 \pm 0.005$ \\
Meander & $0.29 \pm 0.01$ & $2.62 \pm 0.01$ & $0.04 \pm 0.005$ \\
\hline
\end{tabular}

Here $P_{s}$ is the maximal value of reversible polarization in $\mathrm{C} / \mathrm{m}^{2}, P_{\text {irr }}$ is the irreversible component polarization, $\tau$ is the time constant in $s$, characterizing the exponential decay of polarization, and $f$ is the frequency in $\mathrm{Hz}$. The corresponding numerical data determined for sine and meander wave electric field are given in Table 1.

According to Merz [18] full switching reversal of a ferroelectric crystal is only possible when the time of field action exceeds a certain limit $t_{\mathrm{cr}}$. So the time constant may be interpreted as $\tau=T / 2=1 / 2 f_{\mathrm{cr}}$, where $f_{\mathrm{cr}}$ is the maximal frequency for which the full reversal is possible in the given field.

The values of $\tau$ obtained by approximation show that the critical frequency depends not only on the voltage frequency (Figure 6), but also on its shape (Table 1). The maximal experimentally observed $P_{r}$ has a value of $0.64 \cdot P_{s}$ for sine wave and $0.77 \cdot P_{s}$ for meander excitation, thus confirming the effect of the shape, that is, the time of maximum field action on the switching reversal process. It should be taken into account that this inference was drawn for the sample heated to temperatures above $100^{\circ} \mathrm{C}$, when the coercive field of the PZT ceramics is substantially reduced.

Now let us consider the possible causes of the sample self-heating. The mechanisms of dielectric losses may be described in the following way:

$$
E P=\frac{U Q}{d S}=\frac{U I t}{V}=\frac{W t}{V}=\frac{W}{V f}=\frac{Q_{v}}{f},
$$

where $E$ is the intensity of applied field, $P$ is the polarization calculated by dielectric hysteresis loop data, $U$ is the generator voltage, $d$ is the sample thickness, $Q$ is the charge, $S$ is the sample area, $I$ is the switching current passing during the cycle time $t, f$ is the measurement frequency of the AC field, and $V$ is the sample volume. The quantity $Q_{v}=E P f$ is the dissipation power of a sample volume unit. In essence, this energy is responsible for heating the sample.

So, on the one hand, the temperature should be increased with frequency, as it is shown by Figure 4 for sine excitation at $f$ up to $210 \mathrm{~Hz}$. The authors of [16] mentioned this effect. However, on the other hand, during the process of loop formation the initial sharp increase of the switched polarization is replaced by a decrease of the former. This fact is explained by an exclusion of some regions of the sample from the process of switching. It was not taken into consideration in the article [16].

\section{Conclusion}

It follows from the obtained results that the value of switched polarization depends on the AC field amplitude, its shape, and 


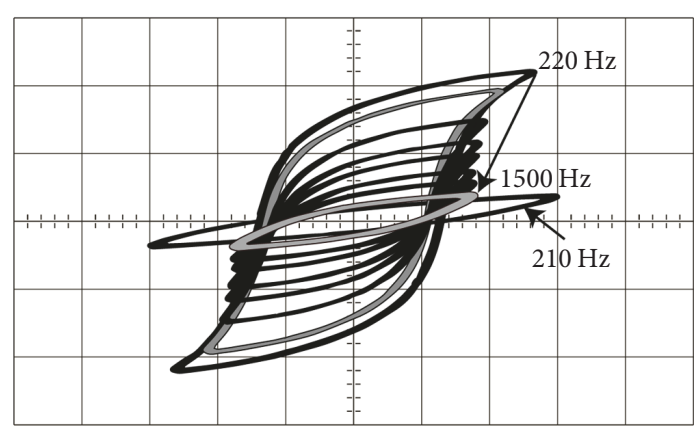

(a)

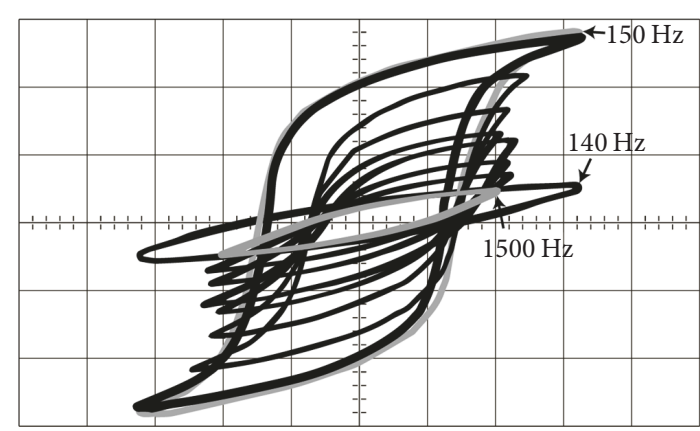

(b)

Figure 5: Frequency dependence of the hysteresis loop shapes in the range of 140 to $1500 \mathrm{~Hz} . E_{m}=840 \mathrm{~V} / \mathrm{mm}$, sample area $25 \mathrm{~mm}{ }^{2}$. (a) Sine wave; (b) meander.

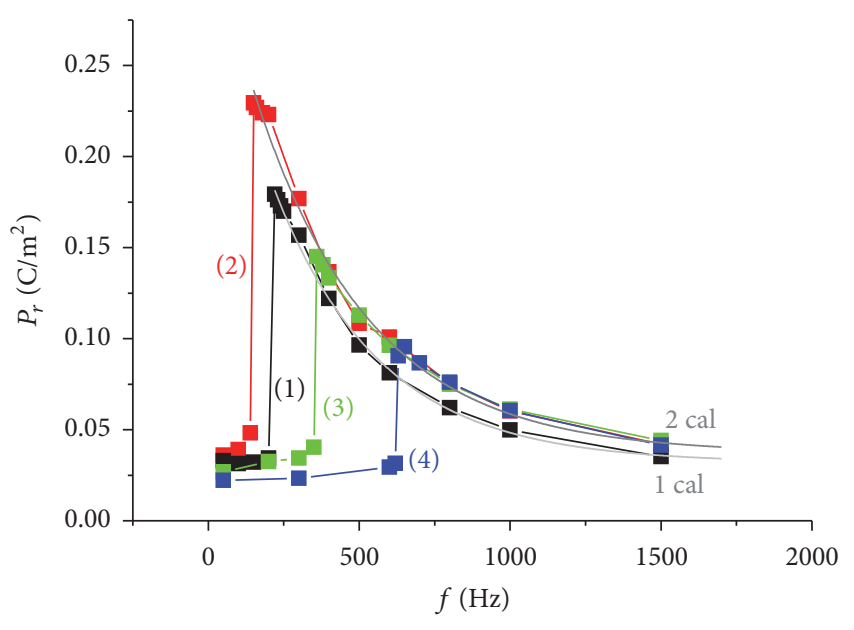

FIGURE 6: Dependence of the switchable polarization value on frequency (curves (2) to (4): experiment, 1 cal and 2 cal: calculation). Curve (1) sine field; (2)-(4) meander; (1), (2) $E_{m}=840 \mathrm{~V} / \mathrm{mm}$; (3) $E_{m}=700 \mathrm{~V} / \mathrm{mm} ;(4) E_{m}=600 \mathrm{~V} / \mathrm{mm}$.

frequency. As this takes place three regions of interest may be separated from the dependence of the maximal switched polarization on frequency (Figure 6).

First one defines the linear rise of the switched polarization at the expense of self-heating of the sample with the increase of the frequency. In this frequency range only minor hysteresis loops are observed.

The second region corresponds to maximal self-heating temperatures (Figure 4). In this case a decrease of the coercive field is evident (due to increasing of the sample temperature). As a result the minor hysteresis loops transform into full ones; the switched polarization is maximal (Figure 6).

Third region is characterized by an exponential decay of the switched polarization with the increase of the frequency. Inasmuch as half-wave time decreases with the increase of the frequency and so does the time of field semiperiodic action, the observed regularity is in full accordance with the theory of Merz according to which switching at a given field value requires definite time. This conclusion is also supported by the dependence of switched polarization on the shape of switching field (Figure 6). At these frequencies the selfheating temperature also starts to decrease (Figure 4).

Decrease of the self-heating temperature with the decrease of switching sample volumes serves as an evidence that the main cause of self-heating in AC fields is the domain wall motion. This inference is supported by the previous data on SBN crystals demonstrating self-heating corresponding to the region of minor hysteresis loops (i.e., region of switching processes) [19]. It is implied that in the present case we consider the fields which initially (at room temperature) are smaller than the coercive ones but exceed the former in the course of the temperature rise of the sample.

Concluding, our results show that the limitations in the domain wall mobility in high-frequency AC fields lead also to restrictions of self-heating process. Therefore it follows that the applicability of formula (2) is limited by the critical frequency value, $f_{\text {cr }}$. Here it should be mentioned that, inasmuch as the self-heating temperature depends directly on the geometrical parameters of the sample [16], the value of $f_{\mathrm{cr}}$ should be determined not only by the material properties but also by the geometry of the sample.

\section{Conflicts of Interest}

The authors declare that they have no conflicts of interest.

\section{Acknowledgments}

The presented work was performed under the State Assignment no. 3.8032.2017/BCh of the Ministry of Science and Education of Russia.

\section{References}

[1] A. Jha, MEMS and nanotechnology-based sensors and devices for communications, medical and aerospace applications, CRC Press, 2008.

[2] N. Jalili and M. Afshari, Piezolectric-based vibration control: from macro to micro in, Nano Scale Systems, Springer, New York, 2010. 
[3] K. Visvanathan and Y. B. Gianchandani, "Microheaters based on ultrasonic actuation of piezoceramic elements," Journal of Micromechanics and Microengineering, vol. 21, no. 8, Article ID 085030, 2011.

[4] K. Uchino and J. R. Giniewicz, Micromechatronics, 2003.

[5] T.-Y. Zhang, M. Zhao, and P. Tong, "Fracture of piezoelectric ceramics," Advances in Applied Mechanics, vol. 38, no. C, pp. 147-289, 2002.

[6] G. Liu, S. Zhang, W. Jiang, and W. Cao, "Losses in ferroelectric materials," Materials Science and Engineering: R: Reports, vol. 89, pp. 1-48, 2015.

[7] S. Ueha, Y. Tomikawa, M. Kurosawa, and N. Nakamura, Ultrasonic Motors: Theory and Applications, Clarendon press, Oxford, UK, 1993.

[8] T. Shigematsu, M. K. Kurosawa, and K. Asai, "Nanometer stepping drives of surface acoustic wave motor," IEEE Transactions on Ultrasonics, Ferroelectrics, and Frequency Control, vol. 50, no. 4, pp. 376-385, 2003.

[9] M. S. Senousy, F. X. Li, D. Mumford, M. Gadala, and R. K. N. D. Rajapakse, "Thermo-electro-mechanical performance of piezoelectric stack actuators for fuel injector applications," $J$. Intell. Mater. Syst. Struct, vol. 20, artuicle 045008, Article ID 045008, 2009.

[10] J. H. Harlow, Electric power transformer engineering, CRC Press, Baco Raton, 2004.

[11] B. Crowell, Vibrations and Waves in. Light and Matter online text series, California, Fullerton, 2006.

[12] M. Tooley, Electronic circuits: fundamentals and applications, Elsevier, 2006.

[13] T. Ikeda, Fundamentals of Piezoelectric Materials Science, Ohm Publication Company.

[14] Q. M. Zhang, H. Wang, and J. Zhao, "Effect of Driving Field and Temperature on the Response Behavior of Ferroelectric Actuator and Sensor Materials," Journal of Intelligent Material Systems and Structures, vol. 6, no. 1, pp. 84-93, 1995.

[15] T. Sakai and H. Kawamoto, "Durability Properties of Piezoelectric Stack Actuator," Japanese Journal of Applied Physics, vol. 137, pp. 5338-5341, 1998.

[16] J. Zheng, S. Takahashi, S. Yoshikawa, K. Uchino, and J. W. C. De Vries, "Heat generation in multilayer piezoelectric actuators," Journal of the American Ceramic Society, vol. 79, no. 12, pp. 31933198, 1996.

[17] O. V. Malyshkina and A. Y. Eliseev, "Power Dissipation during Dielectric Loop Evolution in PZT Ceramics," Ferroelectrics, vol. 480, no. 1, pp. 10-15, 2015.

[18] W. J. Merz, "Domain formation and domain wall motions in ferroelectric $\mathrm{BaTiO}_{3}$ single crystals," Physical Review, vol. 95, no. 3, pp. 690-698, 1954.

[19] O. V. Malyshkina, B. B. Pedko, and V. S. Lisitsin, "Influence of Eu impurity on the dielectric properties of $\mathrm{Sr} x \mathrm{Bal}-\mathrm{xNb} 2 \mathrm{O} 6$ crystals," Crystallography Reports, vol. 60, no. 2, pp. 263-266, 2015. 

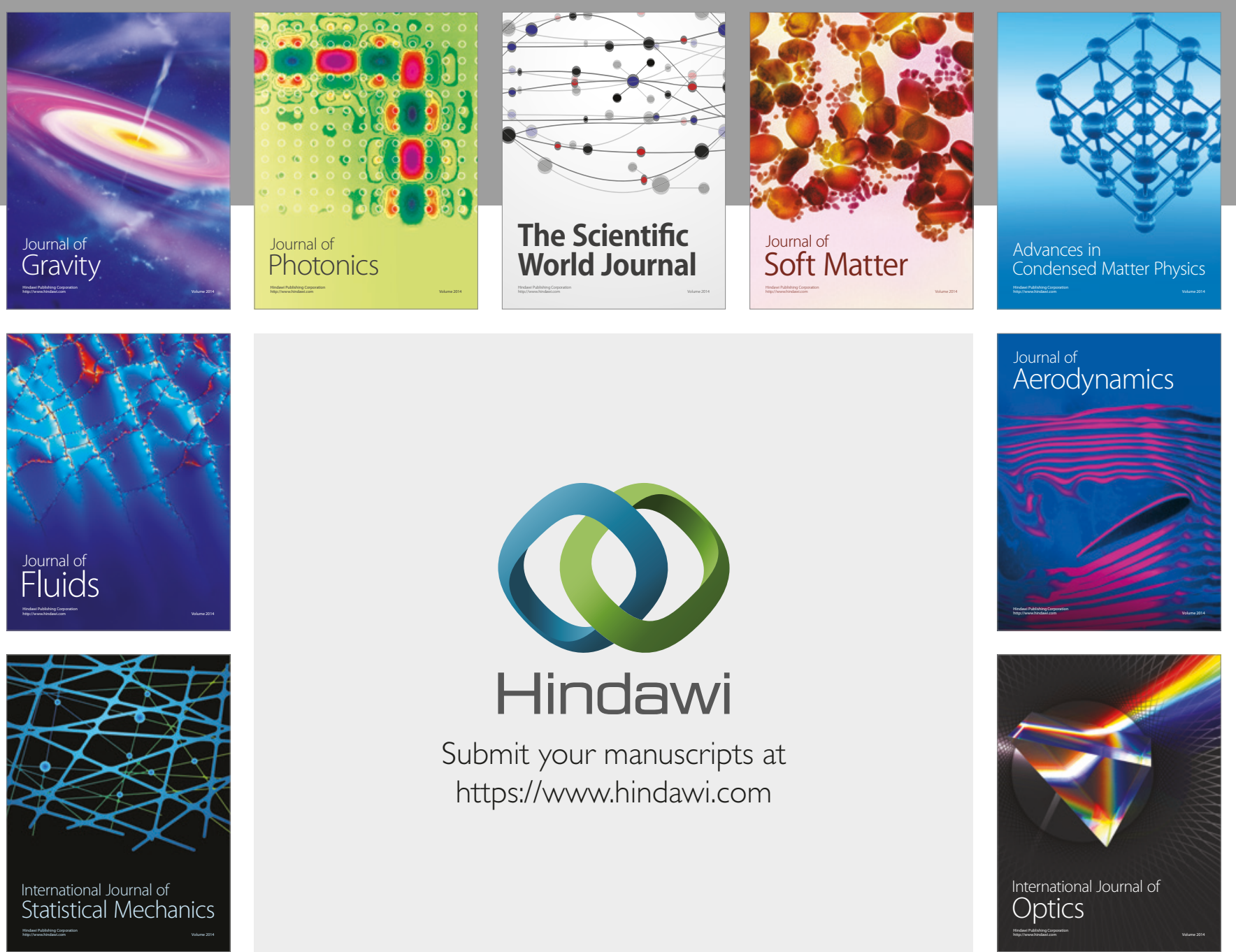

Submit your manuscripts at

https://www.hindawi.com
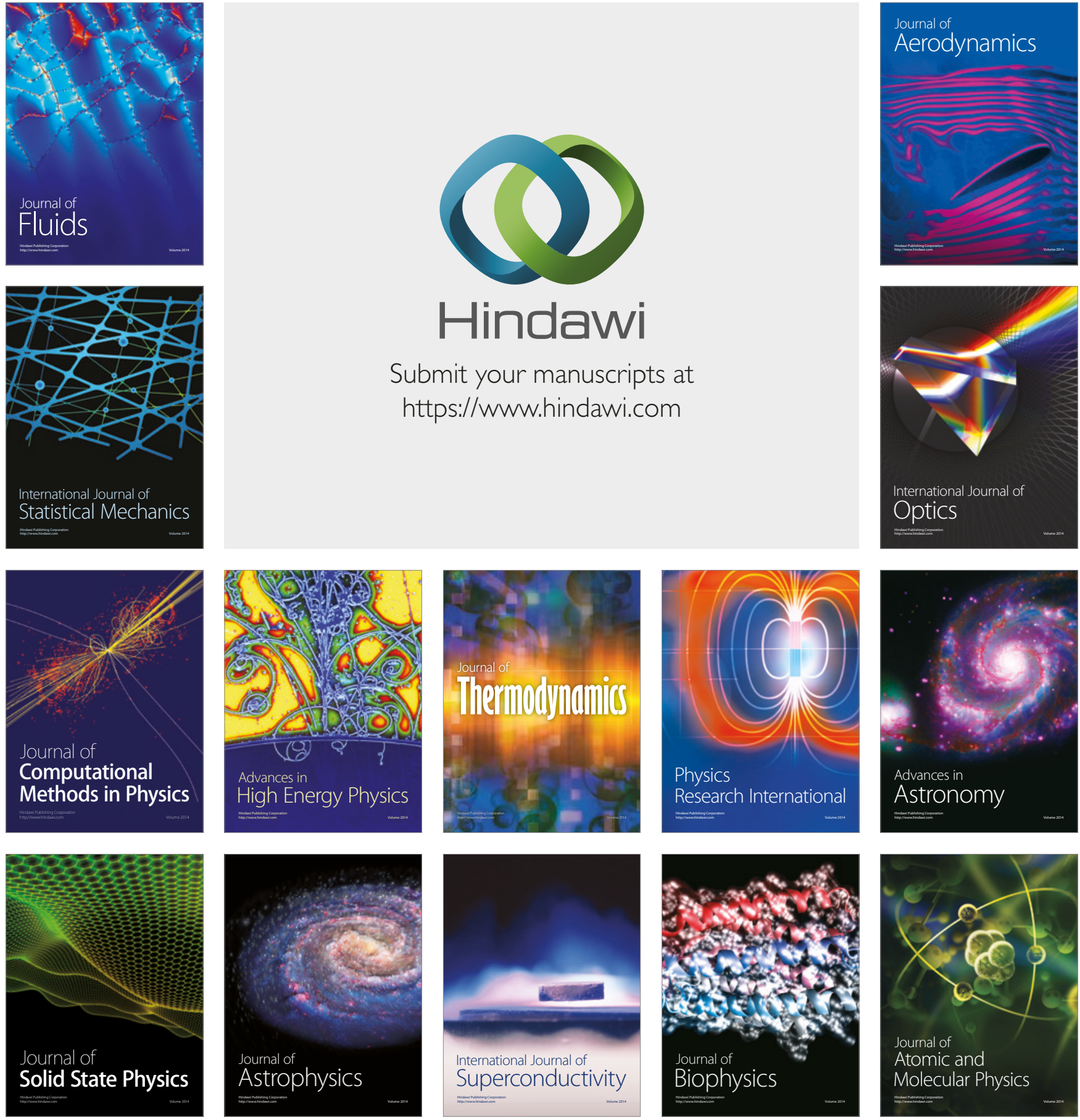